\title{
Immune Therapy of Lympho-Hemopoietic Malignancies
}

\author{
Hakim Echchannaoui ${ }^{a, b}$ Matthias Theobald ${ }^{a, b}$ \\ a University Cancer Center (UCT), Research Center for Immunotherapy (FZI), Department of Hematology, Oncology, and Pneumology, \\ University Medical Center (UMC) of the Johannes Gutenberg-University, Mainz, Germany; \\ ${ }^{b}$ German Cancer Research Consortium (DKTK), partner site Frankfurt/Mainz, Mainz, Germany
}

In recent decades, antibody-based immunotherapy has become a front-line treatment for lympho-hemopoietic malignancies. One of the oldest forms of cancer immunotherapy is allogeneic hemopoietic stem cell transplantation (HSCT). It remains one of the best therapeutic options available for many patients with malignant and non-malignant diseases involving the lympho-hemopoietic system. This is particularly the case for high-risk and relapsed or refractory acute myeloid (AML) and lympoblastic (ALL) leukemia. Nevertheless, disease relapse occurs and graft-versus-host disease (GVHD) as well as post-transplant infectious complications represent main causes for transplant-related morbidity and mortality, thus calling for new treatment options. We are currently witnessing an era of an accelerating development of novel immune therapeutics harnessing the immune system of patients. The aim of this issue of Oncology Research and Treatment is to highlight the most promising categories of immunotherapies in the treatment of lymphohematologic malignancies.

Monoclonal antibodies (mAbs) binding to specific proteins on cancer cells have achieved tremendous success in cancer therapy in the past few years. Rituximab (anti-CD20 mAb) was the first mAb to be approved by the United States Food and Drug Administration (FDA) for the treatment of malignant lymphoma and remains the most frequently used antibody in treatment regimens of a variety of B-cell malignancies. New mAbs directed against CD20, such as ofatumumab and obinutuzumab, have been approved for the treatment of chronic lymphocytic leukemia (CLL) and relapsed/ refractory $(\mathrm{r} / \mathrm{r})$ follicular lymphoma (FL). Several other targets for mAbs, including CD52 (alemtuzumab) and CD40 (dacetuzumab), have been evaluated in clinical studies in CLL, peripheral T-cell lymphoma, and diffuse large B-cell lymphoma (DLBCL). Multiple Myeloma (MM) is targeted by daratumumab (anti-CD38) and elotuzumab (anti-CD319/SLAMF7). New types of antibodies fused to substances which directly kill cancer cells, known as antibody-drug conjugates (ADC), have emerged. Brentuximab vedotin, an ADC to CD30 is approved for the treatment of $r / r$ Hodgkin's lymphoma (HL) and relapsed systemic anaplastic lymphoma. Complete responses have been achieved with ADCs that target CD22 and CD33 in patients with $r / r$ acute ALL and relapsed AML, respectively. Another novel and promising class of immune therapeutics are bispecific T-cell engagers (BiTEs). They are designed to bind malignant cells and attract and activate cytotoxic T lymphocytes. Blinatumomab, which has dual specificity for CD3 and CD19, has shown substantial efficacy in patients with $\mathrm{r} / \mathrm{r}$ B-ALL and DLBCL.

Among novel immunotherapy approaches currently evaluated in patients with lymphoma or hematologic malignancies, immune checkpoint inhibitors, chimeric antigen receptor (CAR) modified $\mathrm{T}$ cells, and vaccines are the most exciting and promising treatment options. In this issue of Oncology Research and Treatment, these current developments are highlighted. Engert and Bröckelmann [1] focus on checkpoint inhibition in HL while Hess [2] provides insight into the current status of these compounds in nonHodgkin's lymphoma (NHL). Advances of cellular immunotherapy of B-cell malignancies are discussed by Schwarzbich and WitzensHarig [3]. Anti-tumor vaccination in hematologic malignancies has been particularly successful by targeting Wilms' tumor protein-derived antigens as outlined by Oka and colleagues [4]. Krackhardt and Audehm [5] give an overview of combining specific adoptive cellular immunotherapy with allogeneic HSCT.

\section{Disclosure Statement}

The authors declare no conflict of interest.

\section{KARGER}

() 2017 S. Karger GmbH, Freiburg

Fax +497614520714 


\section{References}

1 Bröckelmann PJ, Engert A: Checkpoint inhibition in Hodgkin lymphoma - a review. Oncol Res Treat 2017; 40: DOI: $10.1159 / 000481800$.

2 Hess, G: Checkpoint inhibition in non-Hodgkin's lymphoma. Oncol Res Treat 2017; 40: DOI: 10.1159/ 000481888.

3 Schwarzbich MA, Witzens-Harig M: Cellular immunotherapy in B-cell malignancy. Oncol Res Treat 2017; 40: DOI: $10.1159 / 000481946$
4 Oka Y, Tsuboi A, Nakata J, Nishida S, Hosen N, Kumanogoh A, Oji Y, Sugiyama H: Wilms' Tumor Gene 1 (WT1) peptide vaccine therapy for hematological malignancies: from CTL epitope identification to recent progress in clinical studies including a cure-oriented strategy. Oncol Res Treat 2017; 40: DOI: 10.1159/ 000481353
5 Audehm S, Krackhardt AM: Specific adoptive cellular immunotherapy in allogeneic stem cell transplantation. Oncol Res Treat 2017; 40: DOI: 10.1159/ 000484051. 